\title{
The calibration of neutral density wedges
}

CARL R. INGLING, JR., ${ }^{1}$ CENTER FOR VISUAL SCIENCE, UNIVERSITY OF ROCHESTER, Rochester, New York 14627

A method for calibrating neutral-density filters is described.

This article will describe a method for calibrating the type of optical attenuator known as a neutral-density wedge for use in controlling the intensity of monochromatic beams. Neutral-density wedges are widely used in optical stimulators to vary light intensity. Although intensity can be monitored on-line, or measured for every wedge setting by detectors placed in the optical channels, it is customary instead to regulate the lamp and calibrate the wedges in the channels so that the intensity is known from the wedge setting by reference to a calibration curve or table.

Neutral-density wedges have the property that the optical density varies along the length, or around an annulus, of the wedge. The wedges come in either a circular or rectangular configuration, although some materials used for wedges lend themselves to one construction more easily than another. To the author's knowledge, none of the materials from which wedges are made are neutral, or spectrally flat-i.e., the density at a particular point on the wedge is not constant as wavelength is varied. Some of the common materials used in making wedges are listed in Table 1. For wedges that may be expected to follow Bouguer's law, of course the nonneutrality becomes more pronounced at higher densities. For metallic film wedges, the neutrality also becomes worse at higher densities, but it does not follow a pattern predictable from measurements made at lower densities.

In the procedures described below, it is assumed that the wedge is placed in a focal plane conjugate with the source, or behind a small aperture that has the source imaged upon it. This is the usual practice in Maxwellian view systems, since the wedge is then conjugate with the source image in the pupil, and there is no wedging or nonuniformity across the visual field from the wedge gradient, and hence no necessity to counterbalance the wedge gradient with a balancing wedge. When employed this way, only a small strip of the wedge is used. ${ }^{2}$ This is the portion of the wedge that should be calibrated, since there is no assurance that all annular strips on a circular neutral wedge will produce identical calibration curves. Therefore, care should be taken throughout the procedures below to insure that the same radius is used for all the measurements, or the same distance from the edge on rectangular wedges.

\section{METHOD}

Figures 1 and 2 show two forms of calibration curves. A complete calibration of the wedge will consist of a volume with dimensions $\theta$ (angular or linear displacement), $\lambda$ (wavelength), and $D$ (optical density). Figure 1 shows the density as a function of displacement, with wavelength as the parameter; a complete wedge calibration consists of, say, 31 such plots, supposing $10-\mathrm{nm}$ intervals to be chosen. Figure 2 shows $\theta$ as the parameter.

There are a number of ways to generate the curves of Figs. 1 and 2, and once obtained, to reduce the data. I will first describe what has been found to be the most convenient method and then briefly indicate some modifications, which, although more laborious, have some advantages in that simpler equipment is required and the wedge may be calibrated in the apparatus.

(1) Mount the wedge on a simple holder so that $\theta$ can be set and read; see Fig. 3 . The aperture shown in the holder should be the same size as that to be used in the stimulator and positioned at the same radius. Also, the pointer and the aperture must have the same relationship on the holder that they do in the stimulator. An easy way to align the wedge is to set it so that a reference zero line scribed on the clear glass portion of the wedge bisects the aperture when the pointer reads zero (or some criterion). The maximum density-clear glass junction may be used in place of a scribed line.

(2) Place the holder and wedge in a recording spectrophotometer (the author has used a Cary 14 with a large sample chamber) so that the beam is centered upon the aperture. Set the wedge at successive $\theta$ s and measure the density as a function of wavelength at each $\theta$. For a metallic film wedge with a maximum density of 3 , measure about 10-15 curves. Inspection will tell how many; measure enough curves so that differences between successive curves are small. The $\theta$ spacing need not be uniform. These curves are the

Table 1

Common Materials Used in the Manufacture of Neutral Density Wedges. None of the materials listed in this Table can be assumed to be neutral. At densities of 2 and above, all materials in the Table will depart from neutrality by a factor of 2 (0.3 density unit) within the visible spectrum, the particular departure being characteristic of the material.

\begin{tabular}{|c|c|c|c|c|}
\hline Wedge Material & Construction & Stability & Scatter* & Heat Resistance** \\
\hline $\begin{array}{l}\text { Carbon dispersed } \\
\text { in gelatin }\end{array}$ & $\begin{array}{l}\text { Cast in circular } \\
\text { or rectangular molds }\end{array}$ & Stable & No & Must be protected \\
\hline $\begin{array}{l}\text { Metallic film } \\
\text { on glass }\end{array}$ & $\begin{array}{l}\text { Evaporated on } \\
\text { circular or } \\
\text { rectangular blanks }\end{array}$ & Stable & Yes & Very heat resistant \\
\hline Black glass & Rectangular & Stable & Yes & $\begin{array}{l}\text { Heat resistant } \\
\text { (depends on whether } \\
\text { the wedge is } \\
\text { cemented to a blank; } \\
\text { the cement may } \\
\text { part) }\end{array}$ \\
\hline $\begin{array}{l}\text { Photographic film } \\
\text { on neutral base }\end{array}$ & Any shape & $\begin{array}{l}\text { Fairly } \\
\text { stable }\end{array}$ & No & Must be protected \\
\hline $\begin{array}{l}\text { Dyed gelatin and } \\
\text { carbon (Wratten 96) }\end{array}$ & $\begin{array}{l}\text { Cast in circular or } \\
\text { rectangular molds }\end{array}$ & $\begin{array}{l}\text { Fairly } \\
\text { stable }\end{array}$ & Yes & Must be protected \\
\hline Molded plastic & & $\begin{array}{l}\text { Fairly } \\
\text { stable }\end{array}$ & Yes & Must be protected \\
\hline
\end{tabular}

* Entries in thes column indicate if the wedge is recommended for use in an image-forming beam. ** Because the density of optical materials may vary with temperature, it may be well to avoid large temperature changes as a matter of practice. 


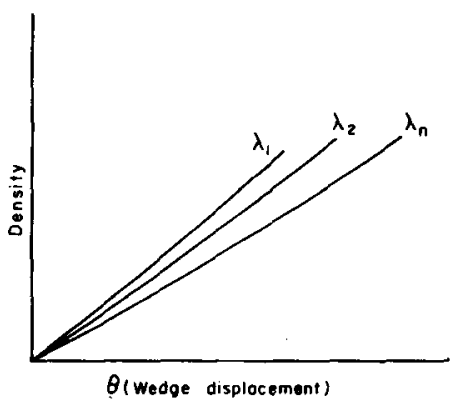

Fig. 1. Density as a function of wedge displacement for given wavelengths. A complete calibration consists of a set of curves for, say, 10-nm intervals from 400 to $700 \mathrm{~nm}$.

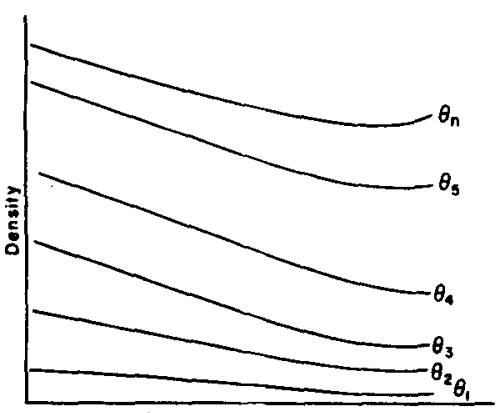

$\lambda$ (Wovelength)

Fig. 2. Density as a function of wavelength for several wedge positions. A complete wedge calibration consists of a series of such spectral traces properly spaced on the density axis. spectral scans shown in Fig. 2, density as a function of wavelength at a given $\theta$.

(3) It is now necessary to measure one curve as illustrated in Fig. 1; see Fig. 4. The measurement of one D vs $\theta$ curve will give the proper spacings for the spectral scans at fixed $\theta$. This is necessary, since, so far, the spectral scans give only relative density. While the spacings between the traces could be measured on the spectrophotometer, it is generally more accurate to measure the spacings on a photometer bench; see Fig. 5. Suppose the lamp is set at $d_{0}$ and the wedge at $\theta_{2}$; note a criterion detector reading. Rotate the wedge to $\theta_{1}$ and adjust the lamp to bring the detector to criterion again. Then $D_{\theta_{1}, \theta_{2}}=2\left(\log d-\log d_{0}\right)$. In this way, the density difference between each $\theta$ can be accurately measured. Since the density steps are small, the range of the bench need not be large. The accuracy can be checked by measuring as large a section of the wedge as the bench will accommodate to see if the summed small sections agree with the density measured for the large section.

(4) Step 3 calibrates the wedge for one wavelength, $\lambda=550$, as shown in Fig. 1. It would be possible, although laborious, to measure such curves at 31 wavelengths. The same data can be obtained by adding a constant to each of the spectral profiles measured in Step 2 so that the density at $550 \mathrm{~nm}$ for each curve is made the same as that indicated by the single D vs $\theta$ curve measured at $550 \mathrm{~nm}$. This produces a set of

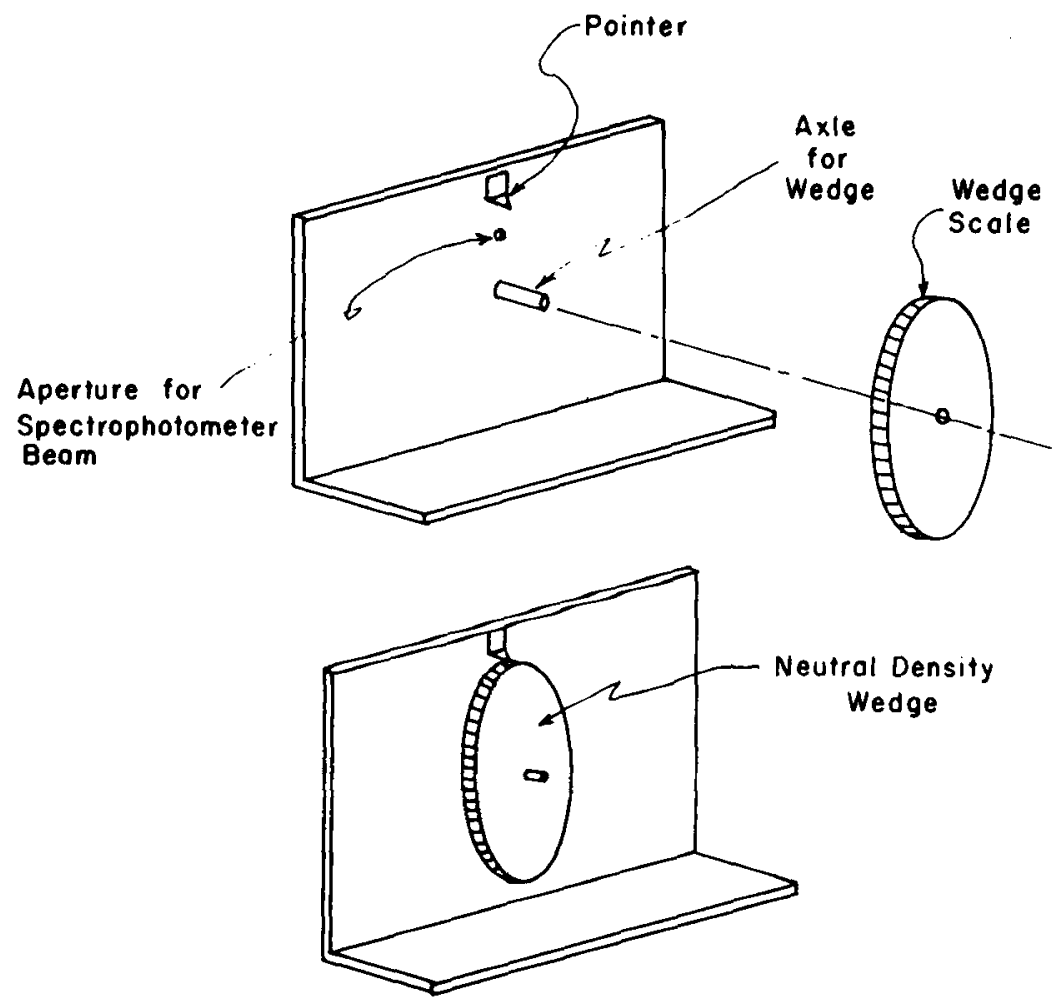

spectral profiles properly spaced as shown in Fig. 4; the relative spectral scans are now absolute. Before proceeding further, one can check Fig. 4 by plotting from it a few $D$ vs $\theta$ curves at selected wavelengths. Such curves should be smooth, with no sharp transitions. If discontinuities appear, more spectral profiles (D vs $\lambda$ ) should be added in the region of the discontinuity (Step 2), and the spacing constants ( $D$ vs $\theta$ ) measured to space them properly in absolute density (Step 3).

(5) The data is now ready for reduction to tables. The author uses a computer program that takes the properly spaced spectral profiles (but see below), extrapolates between the profiles at 10 -nm-wavelength intervals, from 400 to $700 \mathrm{~nm}$ at $1 / 2-\mathrm{deg}$ intervals, and prints out a table for each wavelength; see Table 2. Essentially, these tables give the same data shown in Fig. 1 in tabular form, but the method outlined above for getting the data for computing the tables requires only a fraction of the effort that measuring $31 \mathrm{D}$ vs $\theta$ functions requires; in fact, as outlined, only one such curve need be measured. The program in actual use ${ }^{3}$ accepts as input uncorrected digitized spectrophotometer traces (i.e., traces that have not been properly scaled by a D vs $\theta$ curve) and a set of spacing constants (essentially the same information contained in a $D$ vs $\theta$ curve) and does the spacing before performing the interpolation. Therefore, no hand data reduction is required, other than digitizing the spectrophotometer traces if they are in analog form. The IBM printout is bound and used as the calibration table. To know the correct wedge density at a given wavelength, simply turn to that wavelength page and enter the table with the wedge setting. The present program interpolates to rather fine increments. This is deliberate; it seems preferable to let the computer do the interpolating instead of the table user. Looking a number up in a large table is generally faster and more accurate than interpolating in a small table.

The densities measured in the Cary 14 are specular, or doubly specular, since neither the illumination by nor the collection of the measuring beam is diffuse. If the wedge is used at the focal plane of an optical stimulator (conjugate to the source), the Cary densities will probably be the same as those found in the focal plane; both situations are doubly specular. One difference between the calibration situation and the conditions of use is the angle at which the rays strike the wedge,

Fig. 3. Wedge holder. The exact dimensions will depend upon the geometry of the spectrophotometer it is used with. 


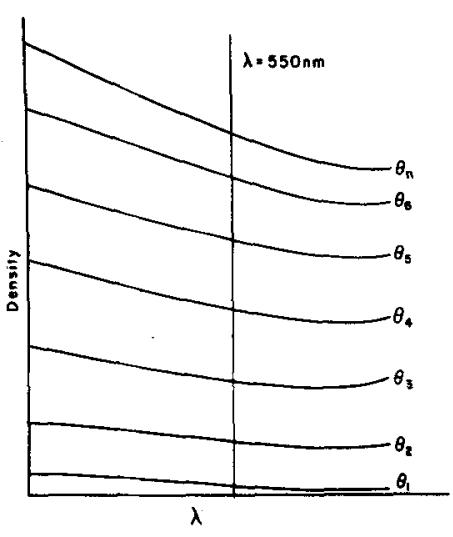

Fig. 4. Proper spacing of spectral profiles measured at various $\theta \mathrm{s}$ by placing them on one $D$ vs $\theta$ curve measured at $\lambda=550 \mathrm{~nm}$.

Table 2

Format for One Page $(\lambda=540 \mathrm{~nm})$ from a Table of 31 Pages $(400-700$, at $10 \mathrm{~nm}$ Intervals) Computed by Interpolation from Fig. 4

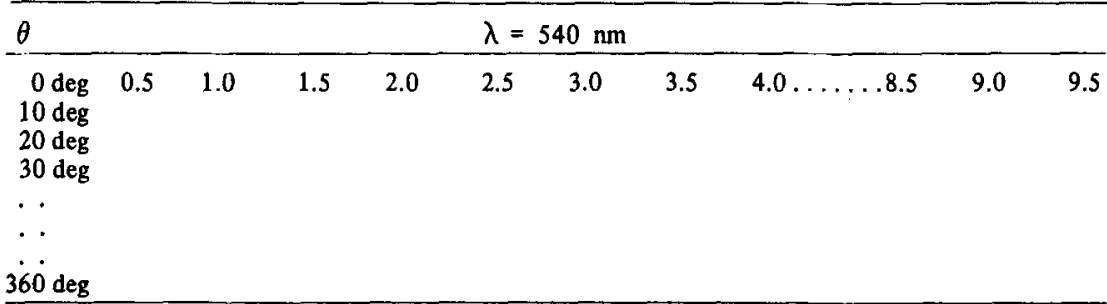

which will be a function of the size of the visual field used in the stimulator. This difference is probably not worth considering; at any rate, it will always be present even if the wedge is calibrated in situ, unless it is calibrated for a fixed visual angle that will not be changed.

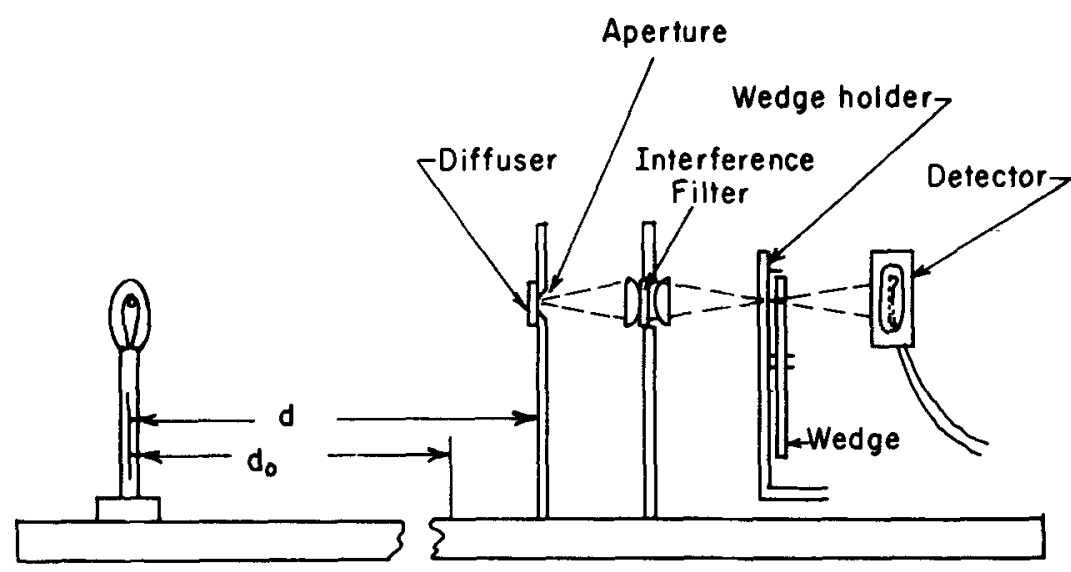

Fig. 5. Arrangement for accurately measuring the density spacing between spectral profiles.

Fig. 6. Set-up for in situ measurement of wedge data required for $\theta \mathrm{D} \lambda$ volume.

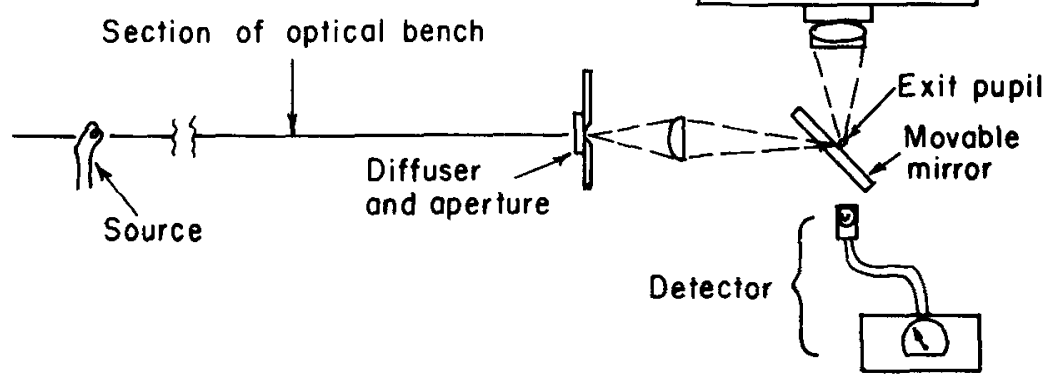

The procedure outlined above produces an accurate $\theta \mathrm{D} \lambda$ volume in table form with a minimum of laboratory measurements, since it relies on an automatic recording spectrophotometer for most of the data, and on a digital computer for interpolation through the volume and preparation of the tables. Assuming the proper equipment is available, once familiarity with the method is gained, a wedge may be completely calibrated and the $\theta D \lambda$ tables be in hand in a matter of a few. hours-in the time it takes for a dozen Cary traces and a dozen bench readings, plus card-punching time. There are, of course, many ways to measure the data required for the computer program to fill the $\theta \mathrm{D} \lambda$ volume. The method briefly outlined below is another and would be used only if more convenient procedures were not feasible, for example, in the case of hardships such as a type of wedge or stimulator design that requires in situ calibration, the detector is not linear, etc.

See Fig. 6. The wedge controls the intensity at the exit pupil. It is assumed that the stimulator contains a device to control wavelength. To measure the spectral profiles, first remove the wedge from the beam or turn it to the clear glass portion. Set $\lambda=400$, say. Position the mirror so that the stimulator beam enters the detector; note the detector reading. Flip the mirror so that the bench beam enters the detector, and adjust the lamp to duplicate the detector reading. Set $\lambda=410$, and repeat, continuing through the spectrum. The curve calculated from the bench readings will be a function of the lamp, transmission of the optics, the monochromator, and the detector sensitivity; i.e., every element in the channel except the neutral-density wedge. Next, rotate the wedge from $\theta_{0}$ (clear glass) to $\theta_{1}$ and repeat the measurements. Subtracting the $\theta_{0}$ trace from the $\theta_{1}$ trace produces a spectral profile (D vs $\lambda$ ) for $\theta_{1}$. Curves for $\theta_{2}, \theta_{3}$, and so on are produced by again subtracting $\theta_{0}$ from subsequent traces. The details of this procedure will not be elaborated, except for the following: in some cases, constraints may exist that will keep the photometer bench 
short (or the reliable range of a linear detector may be short), and it may be found that the range of channel intensities is greater than the range of the bench. In such a case, flatten the channel with a color-balancing filter, which may be left in until all the profiles are measured. For example, a lamp distribution high in the red combined with a photocell response high in the blue may produce a resultant green peak. A magenta filter will flatten this peak and reduce the range of intensities to be accommodated by the bench (or linear detector).
The spacing constants for the profiles are measured by holding wavelength constant $(\lambda=550)$ and measuring densities between the $\theta$ s at which the profiles were measured, again by finding positions of the bench lamp that produce the same deflections as successive wedge positions. Measurement of these densities yields the necessary data for computation of the $\theta \mathrm{D} \lambda$ solid in the manner described previously.

\section{NOTES}

1. Present address: The Institute for Research in Vision, The Ohio State University Research Center, 1314 Kinnear Road, Columbus, Ohio 43212.

2. An intense source (Xenon arc; high wattage quartz-iodine) imaged directly on even a metallic film wedge may, if the wedge is not protected by a heat filter, crack the glass. If such a use is contemplated, a Pyrex substrate is advisable.

3. At the CVS laboratories, University of Rochester, Rochester, N.Y. 14627. The adaptation of the program from a computing center interpolation program was done by Thomas Corwin and John Whiteside.

\section{A geometric method for indexing stimulus relations in association}

\section{RICHARD P. ALLEN, BALTIMORE CITY HOSPITALS, 4940 Eastern Avenue, Baltimore, Maryland 21124}

\begin{abstract}
Methods for indexing stimulus relations in association involve measuring the overlap of the response set and naturally lend themselves to a set-theory method. A geometric method using associative frequencies as distance measures might be suited better to associative data. Two geometric measures are advanced, and it is argued that their accuracy for extreme cases exceeds that for measures from a set-theory approach.
\end{abstract}

One of the current methodological problems in word association involves indexing relations among stimulus words. A recent article by Johnson and Collier (1969) reviewed four indices that have been advanced and suggested two new measures based upon set theoretic considerations. All six indices assume that the relations between stimulus words are best determined by the similarity of responses as indicators of some sort of stimulus equivalence involving semantics or usage within linguistic frameworks. Thus, for a sentence with a word omitted, the likely guesses at the omitted word will be those stimuli with fairly similar responses in a free-association test.

The notion of stimulus equivalence based upon similarity of responses in association seemed a natural condition for the application of set theory to measure the overlap of responses. The article by Johnson and Collier (1969), indeed, comes as the logical conclusion to the approach started by Deese (1962). The use of set theory, however, disregards much of the metric information available from word-association probability that has proved useful in research on perception
(O'Neil, 1953), latencies (Thumb \& Marbe, 1901), and aphasia (Howes, 1965; Allen, 1967). It is proposed in the following paper that a geometric approach provides a measure of stimulus relations better suited to the associative data because it takes account of the metric data and, coincidentally, minimizes the problem of the representational response.

For geometric considerations, each response word can be used as a dimension in a space with the frequency of response indicating the location along the dimension. It is assumed as a first-order approximation that the metric is linear with frequency, the response dimensions are orthogonal, and the dimensions involving each of the stimulus words can be ignored as irrelevant. The distance " $D$ " between two stimulus words is then defined as:

$$
D^{2}=\underset{i}{\Sigma}\left(f_{1, i}-f_{2, i}\right)^{2}
$$

$f_{j, i}=$ the frequency for Response $\mathrm{j}$ to Stimulusj. Defining the metric in probability terms adjusts for sample sizes, thus: $p_{j, i}=f_{j, i} / n_{j}, n_{j}=$ the sample size defined as the number of $S s$ who each gave one association response to the stimulus "j." The linear distance measure, " $\nu$ " now becomes $\nu^{2}=\Sigma\left(\mathrm{p}_{1, \mathrm{i}}-\mathrm{p}_{2, \mathrm{i}}\right)^{2} ;$ if $\mathrm{n}=\mathrm{n}_{1}=\mathrm{n}_{2}$, then $\nu^{2}=1 / \mathrm{n}^{2} \Sigma\left(\mathrm{f}_{1, \mathrm{i}}-\mathrm{f}_{2, \mathrm{j}}\right)^{2}$. As an example, consider that the stimulus "CASE" produced 15 associates of "BOX," 4 of "CARTON," and 1 of "TRIAL," and that the stimulus "CARTON" produced 10 associates of "BOX," 8 of "CASE," and 3 of "PACKAGE," then: $\nu^{2}=$ $(15 / 20-10 / 20)^{2}+(1 / 20-0 / 20)^{2}+$ $(0 / 20-3 / 20)^{2}=0.09$.

Obviously, the assumptions made above are only approximately correct. Since the sum of the response probabilities must be 1.0 , the dimensions are not independent and accordingly not orthogonal. But for large sets of responses, orthogonality seems justified considering the mathematical simplification it introduces. A more important assumption to consider would be the linear metric. The literature reviewed by Allen (1967) demonstrates that the associative frequency metric is approximately logarithmic: the difference between frequencies of 3 and 2 is more like that between 30 and 20 than that between 30 and 29.

Accepting a logarithmic metric poses the serious problem of handling zero frequencies. It is assumed here that a zero frequency may be arbitrarily set at $1 / 2$; this assumption requires, of course, caution since it essentially asserts that, if the sample size were doubled, about half of these zero-frequency responses would occur at least once. The test of this assumption here will be merely the appropriateness of the measure of stimulus relations. The logarithmic distance, " $T$," is: $\mathrm{T}^{2}=\Sigma\left(\log \mathrm{p}_{1, \mathrm{i}}-\log \mathrm{p}_{2, \mathrm{i}}\right)^{2}=\Sigma$ $\log ^{2}\left(p_{1, i} / p_{2}, i\right) ;$ if $n=n_{1}=n_{2}$, then $T^{2}=\Sigma$ $\log ^{2}\left(f_{1, i} / f_{2, i}\right)$. The maximum value of " $T$ " unfortunately depends upon the sample sizes for the stimuli. At least to partially reduce the sample-size complication, the logarithmic geometric measure " $\tau$ " is defined as the ratio of the value of " $T$ " to its maximum value. Thus, $\tau^{2}=\mathrm{T}^{2} / \mathrm{M}^{2}$, $M^{2}=N_{1} \log ^{2}\left(2 \times n_{1} / n_{2}\right)+n_{2}$ $\log ^{2}\left(2 \times n_{2} / n_{1}\right)$, for $n=n_{1}=n_{2} \quad \tau^{2}=$ $\left[1 /\left(2 n \log ^{2} 2\right)\right] \times \Sigma \log ^{2}\left(f_{1, i} / f_{2, i}\right)$, and using common logs, $\tau^{2}=(1 / 0.18 \times n) \times \Sigma$ $\log ^{2}\left(f_{1, i} / f_{2, i}\right)$.

To compare these measures with the six previously advanced, consider the two extreme cases of identical associative responses: Case 1, the two stimuli never elicit each other but have identical 